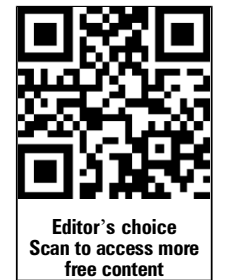

free content
fo access mor
- Additional materials are published online only. To view these files please visit the journal online (http://dx.doi. org/10.1136/gutjnl-2011301373).

For numbered affiliations see end of article

Correspondence to Dr Patrick Tan, Associate Professor, Cancer and Stem Cell Biology, Duke-NUS Graduate Medical School Singapore, 8 College Road, Singapore, 169857: gmstanp@duke-nus.edu.sg

Revised 8 May 2012 Accepted 15 May 2012 Published Online First 26 June 2012

\title{
Comprehensive genomic meta-analysis identifies intra-tumoural stroma as a predictor of survival in patients with gastric cancer
}

Yonghui Wu, ${ }^{1}$ Heike Grabsch, ${ }^{2}$ Tatiana Ivanova, ${ }^{1}$ lain Beehuat Tan, ${ }^{3}$ Jacinta Murray, ${ }^{2}$ Chia Huey Ooi, ${ }^{4}$ Alexander lan Wright, ${ }^{2}$ Nicholas P West, ${ }^{2}$ Gordon G A Hutchins, ${ }^{2}$ Jeanie Wu, ${ }^{1}$ Minghui Lee, ${ }^{1}$ Julian Lee, ${ }^{1}$ Jun Hao Koo, ${ }^{1}$ Khay Guan Yeoh, ${ }^{5}$ Nicole van Grieken, ${ }^{6}$ Bauke Ylstra, ${ }^{6}$ Sun Young Rha, ${ }^{7}$ Jaffer A Ajani, ${ }^{8}$ Jae Ho Cheong, ${ }^{9}$ Sung Hoon Noh, ${ }^{9}$ Kiat Hon Lim, ${ }^{10}$ Alex Boussioutas, ${ }^{11,12}$ Ju-Seog Lee, ${ }^{13}$ Patrick Tan ${ }^{4,14,15}$

\section{ABSTRACT}

Objective Gastric adenocarcinoma (gastric cancer, GC) is a major cause of global cancer mortality. Identifying molecular programmes contributing to $\mathrm{GC}$ patient survival may improve our understanding of $\mathrm{GC}$ pathogenesis, highlight new prognostic factors and reveal novel therapeutic targets. The authors aimed to produce a comprehensive inventory of gene expression programmes expressed in primary GCs, and to identify those expression programmes significantly associated with patient survival.

Design Using a network-modelling approach, the authors performed a large-scale meta-analysis of GC transcriptome data integrating 940 gastric transcriptomes from multiple independent patient cohorts. The authors analysed a training set of 428 GCs and 163 nonmalignant gastric samples, and a validation set of 288 GCs and 61 non-malignant gastric samples.

Results The authors identified 178 gene expression programmes ('modules') expressed in primary GCs, which were associated with distinct biological processes, chromosomal location patterns, cis-regulatory motifs and clinicopathological parameters. Expression of a transforming growth factor $\beta$ (TGF- $\beta$ ) signalling associated 'super-module' of stroma-related genes consistently predicted patient survival in multiple GC validation cohorts. The proportion of intra-tumoural stroma, quantified by morphometry in tissue sections from gastrectomy specimens, was also significantly associated with stromal super-module expression and GC patient survival.

Conclusion Stromal gene expression predicts GC patient survival in multiple independent cohorts, and may be closely related to the intra-tumoural stroma proportion, a specific morphological GC phenotype. These findings suggest that therapeutic approaches targeting the GC stroma may merit evaluation.

\section{INTRODUCTION}

Gastric adenocarcinoma (gastric cancer, GC) is a major cause of global cancer mortality. ${ }^{1}{ }^{2}$ Treatment of GC patients is currently based on clinical parameters such as age, performance status and

\section{Significance of this study}

What is already known on this subject?

- Gene expression profiles of primary cancers can provide 'snapshots' of biological pathways expressed by cancer cells and other cell populations in solid tumours.

- Identifying gene expression programmes associated with patient survival may refine our ability to predict clinical prognosis, discover specific molecular processes regulating disease progression and highlight novel avenues for therapeutic intervention.

\section{What are the new findings?}

- This study reports the largest analysis of gastric cancer transcriptomes to date, providing a comprehensive inventory of expression programmes ('modules') present in gastric cancer.

- Of the 178 modules expressed in primary gastric cancers, expression of a transforming growth factor $\beta$ (TGF- $\beta$ ) associated module of stroma-related genes consistently predicted patient survival in multiple datasets.

- Histopathological analysis of primary gastric cancers revealed that stromal module expression was associated with the proportion of intra-tumoural stroma (ITS).

- Direct histopathological measurement of the ITS proportion was predictive of gastric cancer patient survival.

How might it impact on clinical practice in the foreseeable future?

- Quantifying the proportion of ITS in gastric cancers during routine histopathological assessment may serve as a surrogate marker for stromal gene expression levels in individual tumours.

- Therapies targeting pathways associated with the tumour stroma, such as TGF- $\beta$ signalling, merit consideration in gastric cancer. 
tumour, node, metastasis (TNM) staging, ${ }^{3}$ which are collectively used to decide whether a patient should be treated by surgery alone, surgery plus chemotherapy/chemoradiation or chemotherapy alone. Of these, TNM staging is the major tool used by clinicians to predict GC patient prognosis. However, GC patients with the same TNM stage often exhibit distinct clinical outcomes ${ }^{4}$ suggesting the existence of additional factors influencing GC disease aggressiveness. Previous studies attempting to identify additional GC prognostic factors have investigated a variety of clinical, histological (morphological subtypes, grade of differentiation) and molecular factors, including Ki67 expression (a marker of cell proliferation), p53 mutation status, DNA ploidy and human epidermal growth factor receptor 2 (HER2) expression. $^{5-9}$ However, clear prognostic roles have not been consistently observed for most of these factors, particularly when tested in multiple independent patient cohorts. So far, prognostic studies in GC have focused primarily on aberrations occurring in the GC cell. However, like all solid tumours, GCs are highly complex entities composed of neoplastic epithelial cells, as well as of vessels, fibroblasts, immune cells and extracellular matrix. Compared with cancer cells, relatively little is known regarding the potential contribution of these other components to GC patient prognosis. ${ }^{10-13}$

Gene expression profiling represents a powerful technology that can provide an unbiased and holistic 'molecular snapshot' of distinct gene expression programmes present within a tumour. ${ }^{14}$ While some of the genes comprising these expression programmes are expressed exclusively by cancer cells, other cell types within a tumour may also contribute to the gene expression profile. Here, we hypothesised that a detailed analysis of a large number of GC transcriptomes would provide a comprehensive inventory of distinct expression programmes associated with GC, which can then be tested for associations with patient prognosis. Analysing gene expression data from 940 GCs and non-malignant gastric tissues from different patient populations, we found that the expression of a 'stromal module' comprising stroma-related genes was associated with both, transforming growth factor $\beta$ (TGF- $\beta$ ) signalling and patient survival in multiple GC cohorts. In GC tissue sections, we further found that the proportion of the stroma within GCs (intra-tumoural stroma, ITS) also predicted patient survival. To our knowledge, this is the first study demonstrating a prognostic role for both, stromal gene expression and the ITS proportion in GC patients. Our results highlight the potential role of the ITS proportion as a predictive biomarker to identify subgroups of patients with GCs that might respond to tumour stroma-directed therapies. Moreover, because our molecular analysis indicates that the GC stromal module is associated with TGF- $\beta$ signalling, molecular therapies targeting the TGF- $\beta$ pathway may merit evaluation in GC patients.

\section{METHODS}

\section{Gastric cancer datasets}

Discovery datasets

The GC coexpression network was generated using nine independent GC expression microarray datasets (supplemental table 1), comprising 591 gastric samples (428 GCs and 163 nonmalignant samples (normal gastric mucosa, chronic gastritis, atrophic gastritis with intestinal metaplasia)). One hundred and nine of the 163 non-malignant gastric samples were matched to GC samples, while the remaining 54 non-malignant samples were gastric biopsies from individuals with Helicobacter pylori gastritis without cancer, recruited into a randomised, placebocontrolled trial of $H$ pylori therapy. ${ }^{15}$ The datasets were obtained from the National Center for Biotechnology Information (NCBI) Gene Expression Omnibus (http://www. ncbi.nlm.nih.gov/geo/), or from collaborators (SG-2, LS-1 and AMS). Several of these datasets have been previously published. ${ }^{15-20}$ Detailed clinical information from the discovery datasets was not used in this study, with the exception of the AMS cohort (34 GCs) which was analysed to provide additional power for the survival analysis.

\section{Validation datasets (transcriptome)}

Three GC datasets (SG-3, AU-2 and YGC) were used for validation analyses comprising $288 \mathrm{GC}$ and 61 non-malignant gastric samples. These samples were not used in the discovery phase. All 61 non-malignant validation samples were matched with a GC. Seven patients received neo-adjuvant therapy (one patient, SG-3 cohort; six patients, AU-2 cohort). Clinical characteristics are presented in table 1. Primary GCs were collected with patient consent from the participating centre's tissue repositories or pathology archives and approval from the respective institutional research ethics review committees in accordance with local regulations and legislations. Clinical information was collected with the approval of the Institutional Review Board. Gene expression data of these validation datasets have been deposited under Gene Expression Omnibus accession numbers GSE15459 (SG-3), GSE35809 (AU-2) and GSE13861 (YGC). ${ }^{21}$

\section{Validation dataset (tissue microarray (TMA))}

An additional GC TMA dataset (LS-2) was also analysed. TMAs were constructed from an independent set of 163 GC patients who underwent curative D2 resection at the Academic Department of Surgery, Leeds General Infirmary, Leeds, UK, between 1970 and 1991. After excluding technical failures and data from patients who died within 30 days after surgery (postoperative mortality), results from 131 patients were available for analyses. Median follow-up time after surgery was 5.5 years ranging from 0.11 years to 20.6 years. Forty-nine $(37.4 \%)$ patients had died due to cancer at the end of the study period. Clinical characteristics of the LS-2 TMA dataset are presented in the online supplemental table 2 .

The supplemental methods provide details of GC network construction, functional annotation of network modules, mapping of modules and oncogenic pathways to individual samples, clinicopathological and survival analyses, and quantitation of ITS by computerised point counting.

\section{RESULTS \\ Network analysis identifies multiple conserved gene expression modules in GC}

We established a discovery series of 591 gastric tissue samples (428 cancers and 163 matched non-neoplastic samples), drawn from nine independent GC transcriptome datasets representing a wide variety of GC patient populations from different countries, including countries with low and high GC incidence (online supplemental table 1). We used these discovery datasets to construct a GC gene coexpression network. First, we established a core network of genes commonly present in all nine datasets comprising genes exhibiting consistent and robust expression correlations in both, GCs and non-malignant gastric samples (see Methods, figure 1A). Second, to increase the number of genes in the network, we expanded the network construction to all possible combinations of eight, seven and six datasets. This network construction method yielded a final GC coexpression network comprising 3177 genes linked by 14965 
Table 1 Clinical characteristics of validation datasets

\begin{tabular}{|c|c|c|c|c|c|}
\hline Clinical characteristics & Category & SG-3 n (\%) & AU-2 n (\%) & YGC n (\%) & Total n (\%) \\
\hline & Total & $153(100)$ & $70(100)$ & $65(100)$ & $288(100)$ \\
\hline Gender & $\begin{array}{l}\text { Male } \\
\text { Female } \\
\text { Unknown }\end{array}$ & $\begin{array}{c}95(62.1) \\
53(34.6) \\
5(3.3)\end{array}$ & $\begin{array}{l}48(68.6) \\
22(31.4) \\
0(0)\end{array}$ & $\begin{array}{l}46(70.8) \\
19(29.2) \\
0(0)\end{array}$ & $\begin{aligned} 189 & (65.6) \\
94 & (32.6) \\
5 & (1.7)\end{aligned}$ \\
\hline Age & Median years ( $\min , \max$ ) & $65(23,92)$ & $67(32,85)$ & $63(32,83)$ & $65(23,92)$ \\
\hline TNM stage & $\begin{array}{l}\text { I } \\
\text { II } \\
\text { III } \\
\text { IV } \\
\text { Unknown }\end{array}$ & $\begin{aligned} 27 & (17.6) \\
25 & (16.3) \\
56 & (36.6) \\
42 & (27.5) \\
3 & (2)\end{aligned}$ & $\begin{aligned} 13 & (18.6) \\
16 & (22.9) \\
33 & (47.1) \\
7 & (10) \\
1 & (1.4)\end{aligned}$ & $\begin{aligned} 12 & (18.5) \\
2 & (3.1) \\
35 & (53.8) \\
16 & (24.6) \\
0 & (0)\end{aligned}$ & $\begin{aligned} 52 & (18.1) \\
43 & (14.9) \\
124 & (43.1) \\
65 & (22.6) \\
4 & (1.4)\end{aligned}$ \\
\hline Laurén class & $\begin{array}{l}\text { Intestinal-type } \\
\text { Diffuse-type } \\
\text { Mixed/unclassifiable }\end{array}$ & $\begin{array}{l}72(47.1) \\
59(38.6) \\
22(14.4)\end{array}$ & $\begin{array}{c}34(48.6) \\
30(42.9) \\
6(8.6)\end{array}$ & $\begin{array}{l}20(30.8) \\
31(47.7) \\
14(21.5)\end{array}$ & $\begin{array}{r}126(43.8) \\
120(41.7) \\
42(14.6)\end{array}$ \\
\hline Grade of differentiation & $\begin{array}{l}\text { Well } \\
\text { Moderate } \\
\text { Poor } \\
\text { Undifferentiated/unknown }\end{array}$ & $\begin{aligned} & 4(2.6) \\
51 & (33.3) \\
91 & (59.5) \\
7 & (4.6)\end{aligned}$ & $\begin{array}{cl}2 & (2.9) \\
22 & (31.4) \\
26 & (37.1) \\
20 & (28.6)\end{array}$ & $\begin{array}{l}\text { ND } \\
\text { ND } \\
\text { ND } \\
65(100)\end{array}$ & $\begin{aligned} & 6(2.1) \\
& 73(25.3) \\
& 117(40.6) \\
& 92(31.9)\end{aligned}$ \\
\hline Resection margin status & $\begin{array}{l}\text { Negative } \\
\text { Positive } \\
\text { Unknown }\end{array}$ & $\begin{aligned} & 110(71.9) \\
& 17(11.1) \\
& 26(17)\end{aligned}$ & $\begin{array}{c}66(94.3) \\
4(5.7) \\
0(0)\end{array}$ & $\begin{array}{l}\text { ND } \\
\text { ND } \\
65(100)\end{array}$ & $\begin{aligned} 176 & (61.1) \\
21 & (7.3) \\
91 & (31.6)\end{aligned}$ \\
\hline Non-malignant gastric samples & Matched to GC & 38 & 9 & 14 & 61 \\
\hline
\end{tabular}

interactions (false discovery rate (FDR) $<0.001$, supplemental table 3 , figure $1 \mathrm{~A}$ ).

To partition the network into meaningful and biologically relevant subunits, we then defined 'modules' within the coexpression network, defined as groups of genes showing a high degree of internal coexpression relative to genes external to the group. Using a previously described module construction algorithm, ${ }^{22}$ we identified 178 coexpression modules in the network comprising 10-200 genes each (supplemental figure 1, supplemental document 1 ; supplemental table 4 presents a representative module). Supporting the biological relevance of the GC network, network topology analyses at multiple levels, for example, between (1) all individual genes in the entire coexpression network, (2) all modules and (3) genes within a particular module revealed that the identified networks all exhibited a 'scale-free' structure with most genes acting as 'edges', and certain genes acting as 'hubs' (supplemental figure 2 and supplemental table 5; also see Discussion). These findings are consistent with previous studies establishing that biological networks are often scale-free. ${ }^{23}$

Since coexpressed genes typically act within the same pathway or share related biological functions, ${ }^{24}$ we proceeded to systematically annotate the 178 modules in the coexpression network by comparing their gene content against the Molecular Signatures Database (MSigDB), a publicly accessible database of gene sets annotated by chromosomal position, pathway components, gene ontologies and cis-regulatory binding sites (figure 1B). A total of 148 (83\%) of 178 modules were successfully mapped to at least one MSigDB dataset at the threshold level of significance $(p<0.001$, minimum overlap $>5$ genes), while 30 modules lacked MSigDB assignments and may be novel. Several modules shared similar MSigDB annotations raising the possibility that these modules might participate in common biological programmes. We called these higher order associations 'supermodules', and identified seven distinct super-modules in the coexpression network. One super-module comprised 32 modules related to various aspects of cell cycle and proliferation. ${ }^{25}$ A second super-module, designated the 'stromal super-module', contained 23 modules associated with extracellular matrix biology and stromal cells. The remaining supermodules in the coexpression network were associated with immune response, digestive function, mitochondrial, ribosomal and proteasomal function (see colour legend in figure 1B). Supplemental document 1 provides all the member genes of the 178 modules, and supplemental document 2 provides a comprehensive table of the 178 modules and their MSigDB assignments.

\section{Determining levels of module expression in individual validation samples}

To compare the expression levels of different modules between individual GCs, we used a previously published algorithm (GENOMICA, see Methods and Segal et al, 2004 ${ }^{26}$ ) to map the 178 expression modules onto three independent GC datasets not used in the network construction described above (Singapore dataset: SG-3, 153 GCs and 38 matched non-malignant gastric samples; Australia dataset: AU-2, 70 GCs and 9 non-malignant samples; South Korea dataset: YGC, 65 GCs and 14 matched non-malignant samples). Table 1 provides the clinicopathological characteristics of these datasets. To maximise statistical power, we combined all samples from these datasets (SG-3, AU-2 and YGC) resulting in a combined validation series of 349 samples (288 GCs and 61 non-malignant gastric samples).

Mapping of the expression modules to this combined validation series confirmed that the modules were differentially expressed across individual GCs (figure 2A). We noted interesting relationships between modules. For example, GCs with high expression of cell proliferation modules tended to coexpress modules related to digestive function, while GCs with high expression of the stromal module exhibited low expression of cell proliferation modules and low expression of modules related to digestive function. A subset of GCs showed high expression of modules related to proteosomal function (see Discussion). 

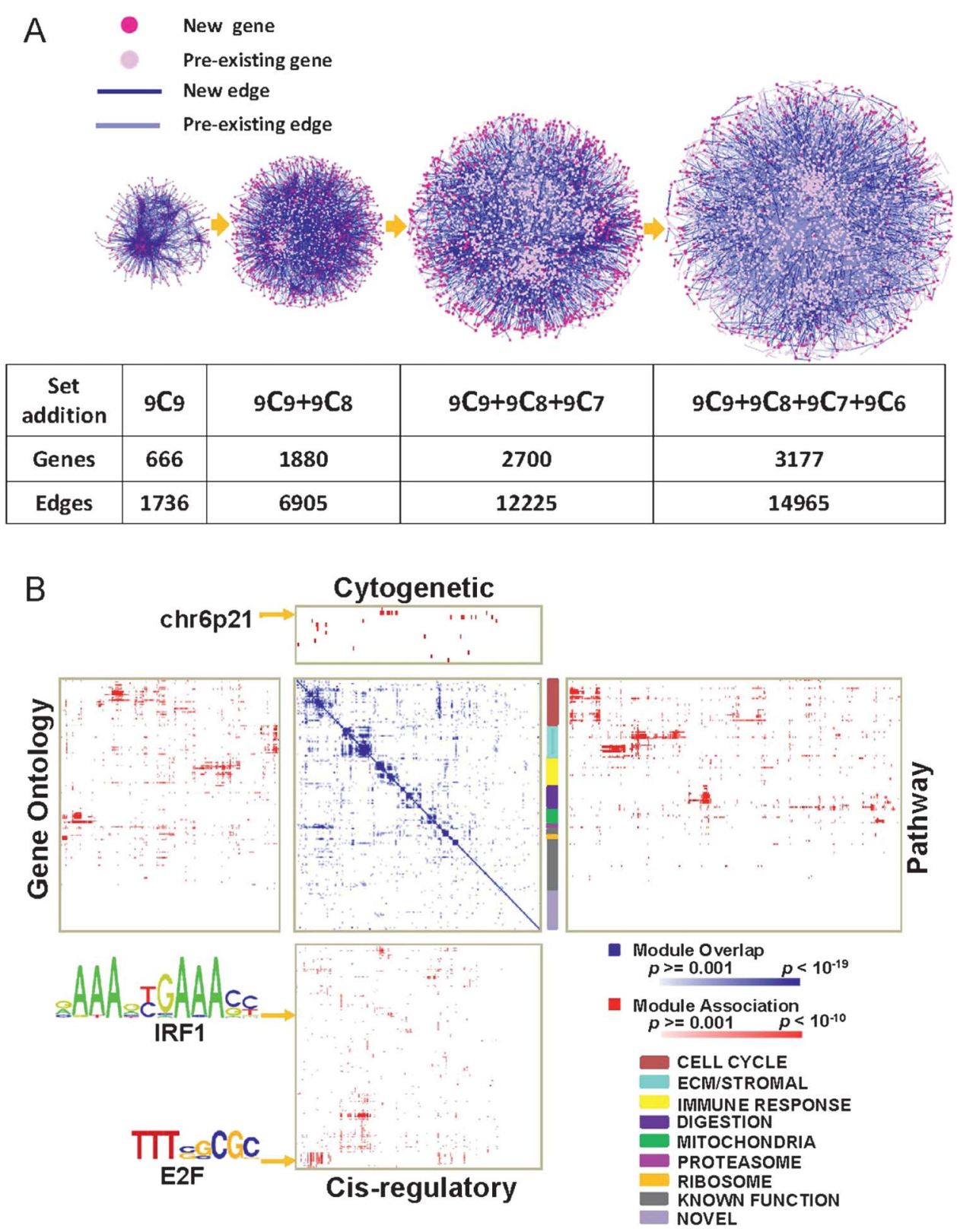

Figure 1 Gastric cancer (GC) coexpression network construction and annotation. (A) Network construction. (left to right) Core network exhibiting conserved coexpression correlations across $9 \mathrm{GC}$ datasets $\left({ }^{9} \mathrm{C}_{9}\right)$. Genes are nodes (pink) and edges are coexpression correlations (blue). The core network was expanded by adding nodes and edges across all possible combinations of 8,7 and $6 \mathrm{GC}$ datasets $\left({ }^{9} \mathrm{C}_{8},{ }^{9} \mathrm{C}_{7},{ }^{9} \mathrm{C}_{6}\right)$. Light pink nodes represent pre-existing nodes already present in the prior network, while dark pink nodes represent new genes. Light blue lines represent pre-existing edges from the prior network, while dark blue lines represent new edges. All genes and edges are associated with a false discovery rate $<0.001$. (B) Functional annotations of modules. (blue-white heat map) Gene composition overlap between the 178 modules. Darker blue regions represent modules with significant gene overlap. The white-blue scale bar indicates $p$ values for the module overlap (hypergeometric test). (Surrounding four red matrices) Module mapping against the Molecular Signatures Database (MSigDB); cytogenetic location (top), pathway signatures (right), cis-regulatory binding sites (bottom) and gene ontologies (left). Darker red areas represent significant module associations (minimum overlap number of genes $=5$ ). The white-red scale bar indicates the $p$ values for the module association. The multicoloured vertical colour bar to the right of the white-blue heat map represents groups of modules exhibiting significant overlap in gene content (super-modules, see colour code at bottom right). Arrows indicate representative cis-regulatory motifs (E2F, IRF1) and chromosomal bands (6p21). E2F- and IRF1-binding motifs are shown using standard position-weight matrix terminology.

\section{GC expression modules are associated with distinct clinicopathological characteristics}

We used the combined validation series to explore if expression of any of these modules might be related to clinicopathological characteristics including age, gender, disease stage, histopathological subtype and grade of differentiation (figure 2A and supplemental table 6). The results from the combined validation series are presented in the paragraphs below and in supplemental table 6, while the results from analyses of the individual datasets can be found in supplemental figure 3 and supplemental table 7 . For these and all subsequent analyses, $\mathrm{p}$ values were corrected for multiple hypotheses testing, and a corrected $\mathrm{p}$ value $<0.05$ was considered significant. 

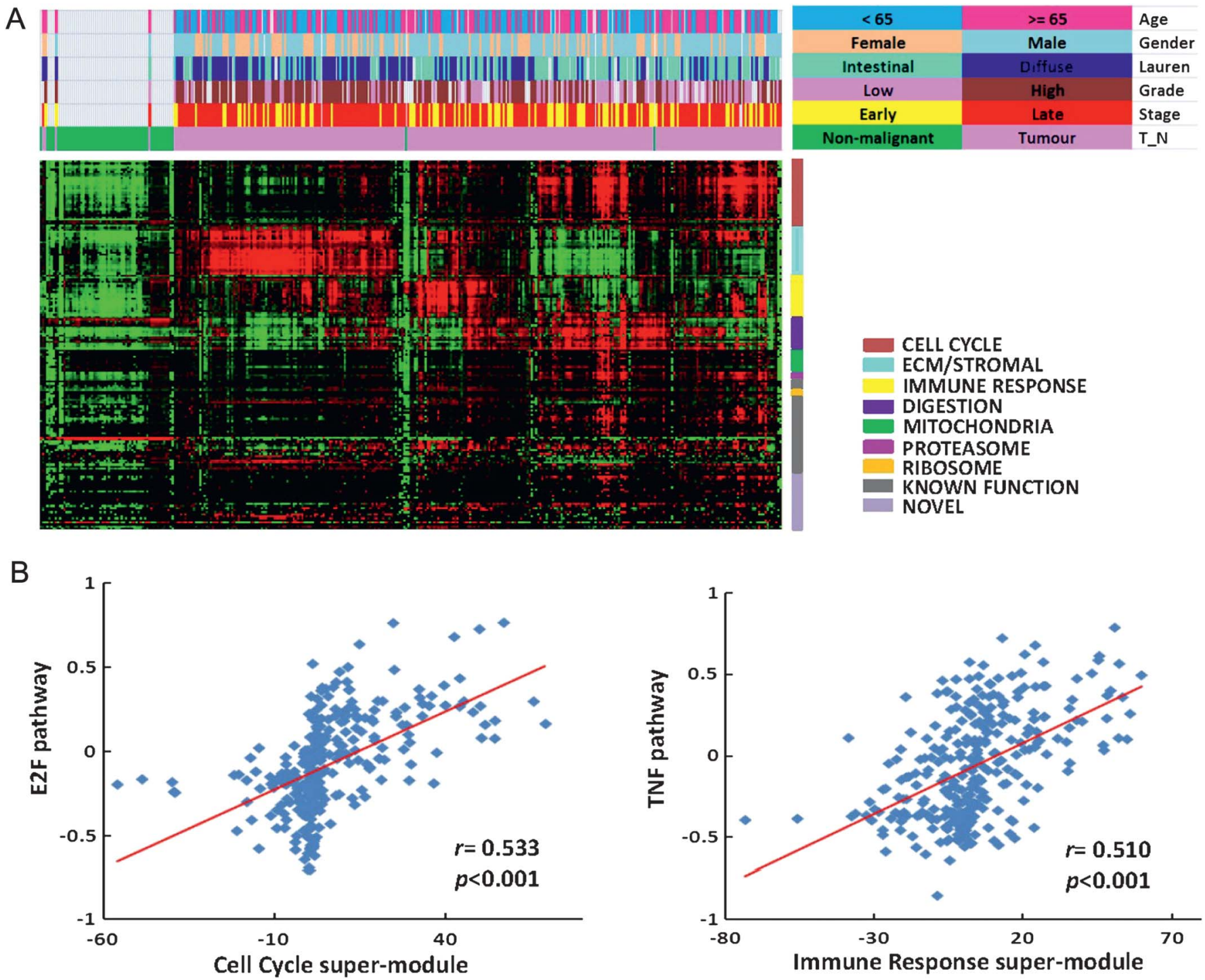

Figure 2 Patterns of module expression are associated with distinct clinicopathological characteristics and oncogenic pathways. (A) The lower red-green heat map represents different patterns of module expression in the combined validation series SG-3/AU-2/YGC dataset (349 samples) ordered by hierarchical clustering. Red represents modules that are highly expressed, while green represents modules expressed at low levels. Coloured bars above the heat map represent clinicopathological characteristics (age, gender, Laurén classification, grade of differentiation, TNM stage, gastric cancer or non-malignant gastric tissue) (colour legend on top right). (B) Oncogenic pathways associated with modules and super-modules. Data originates from the 288 gastric cancers in the 349 combined validation series: SG-3 $(n=153), A U-2(n=70)$ and YGC ( $n=65)$. (Left) E2F pathway activation was significantly correlated with cell-cycle super-module expression (correlation coefficient $r=0.533, p<0.001$ ). The $y$ axis represents levels of E2F pathway activation, while the $x$ axis represents levels of cell-cycle super-module expression. Each data point represents an independent validation sample. (Right) Tumour necrosis factor (TNF) pathway activation correlated with immune response super-module expression (correlation coefficient $r=0.510, p<0.001$ ). The y axis represents levels of TNF pathway activation, while the $x$ axis represents levels of immune response super-module expression.

\section{Age at diagnosis}

GCs from patients younger than 65 years of age exhibited higher stromal and immune response super-module expression (stromal: $\mathrm{p}<1.00 \times 10^{-323}$; immune response: $\mathrm{p}=6.69 \times 10^{-3}$ ), while GCs from patients older than 65 years of age exhibited higher cell cycle $\left(p=1.47 \times 10^{-7}\right)$, mitochondrial $\left(p=4.99 \times 10^{-3}\right)$ and ribosomal $(p=0.028)$ super-module expression.

Gender

GCs from male patients were associated with higher cell cycle $\left(\mathrm{p}<1.00 \times 10^{-323}\right)$ and proteasomal $\left(\mathrm{p}=9.1 \times 10^{-4}\right)$ supermodule expression, while GCs from female patients were associated with higher stromal super-module expression $\left(\mathrm{p}<1.00 \times 10^{-323}\right)$.
Disease stage (UICC (Union for International Cancer Control)/ AJCC (American Joint Committee on Cancer) 6th edition)

Early-stage (stages I and II) GCs were associated with higher cell cycle $\left(p=4.32 \times 10^{-9}\right)$, mitochondrial $\left(p=7.68 \times 10^{-9}\right)$, ribosomal $\left(p=5.87 \times 10^{-3}\right)$ and proteasomal $(p=0.035)$ super-module expression, while late-stage (stages III and IV) GCs were associated with higher stromal super-module expression $(\mathrm{p}<1.00$ $\left.\times 10^{-323}\right)$.

Histopathological subtype

Intestinal-type GCs exhibited higher cell cycle $(p<1.00$ $\left.\times 10^{-323}\right)$, proteasomal $\left(\mathrm{p}=4.95 \times 10^{-14}\right)$, digestive function $\left(\mathrm{p}=6.97 \times 10^{-13}\right)$, mitochondrial $\left(\mathrm{p}=3.0 \times 10^{-3}\right)$ and immune response super-module expression $\left(p=3.45 \times 10^{-3}\right)$, while 
diffuse-type GCs were associated with higher stoma supermodule expression $\left(\mathrm{p}<1.00 \times 10^{-323}\right)$.

\section{Grade of differentiation}

Well and moderately differentiated, low-grade GCs exhibited higher cell cycle $\left(\mathrm{p}<1.00 \times 10^{-323}\right)$, digestive function $\left(\mathrm{p}<1.00 \times 10^{-323}\right)$, mitochondrial $\left(\mathrm{p}=2.93 \times 0^{-9}\right)$ and proteasomal $\left(p=5.49 \times 10^{-7}\right)$ super-module expression, while poorly differentiated and undifferentiated, high-grade GCs were associated with higher stromal $\left(\mathrm{p}<1.00 \times 10^{-323}\right)$ and immune response $\left(\mathrm{p}=1.08 \times 10^{-5}\right)$ super-module expression. We also considered the current WHO guideline that differentiation should only be graded in intestinal-type GCs. ${ }^{27}$ When our analysis was restricted to intestinal-type GCs, low-grade GCs exhibited higher digestive function $\left(p=5.10 \times 10^{-7}\right)$ and mitochondrial $(p=0.002)$ supermodule expression, while high-grade GCs were associated with higher immune response $\left(\mathrm{p}=1.20 \times 10^{-15}\right)$ and stromal $(p=0.027)$ super-module expression.

Supporting the robustness of the above associations, similar trends were also observed for the vast majority of these relationships (61 of 69) when the three independent validation datasets (SG-3, AU-2 and YGC) were analysed individually. Only eight relationships showed an opposite trend in the individual sets compared with the combined 349-sample set, and of these eight, only one was significant (Laurén classification and cellcycle module expression in YGC cohort, $\mathrm{p}$ value $<0.05$, supplemental table 7).

\section{GC expression modules are associated with distinct oncogenic pathways}

Besides investigating the association with clinicopathological characteristics, we also sought to link the expression of the 178 modules to molecular signalling pathways known to be activated or deregulated in malignant tumours. Using a previously described pathway mapping approach, we mapped specific gene expression signatures ('pathway signatures') representing 21 oncogenic and tumour suppressor pathways previously implicated in gastric carcinogenesis (p53, EGFR, TGF- $\beta$, STAT3, VEGF, CEBP, AKT, BRCA1, HER2, SRC, E2F, TNF, MYC, WNT, NF-kB, PI3K, PPARG, p63, RAS, CD31 and HSP90 ${ }^{28-}$ ${ }^{30}$ ) onto the combined validation series. We then identified specific GC modules whose expression was significantly correlated to the expression of the pathway signatures (online supplemental tables 8 and 9).

E2F is a transcriptional regulator of cell-cycle genes. ${ }^{31}$ We found that GCs expressing high levels of E2F pathway activation also expressed 32 distinct cell-cycle-related modules $(r=0.533$, $\mathrm{p}<0.001$; figure 2B, left panel). Supporting E2F as a transcriptional regulator of these modules, a promoter analysis of genes in these cell-cycle-related modules revealed that they were significantly enriched in E2F binding motifs $\left(p=2.41 \times 10^{-7}\right.$; figure $3 \mathrm{~A}$ ).

GCs exhibiting high levels of Tumour Necrosis Factor (TNF) and Nuclear Factor Kappa-light-chain-enhancer of activated B cells (NF-kB) pathway activation also expressed multiple

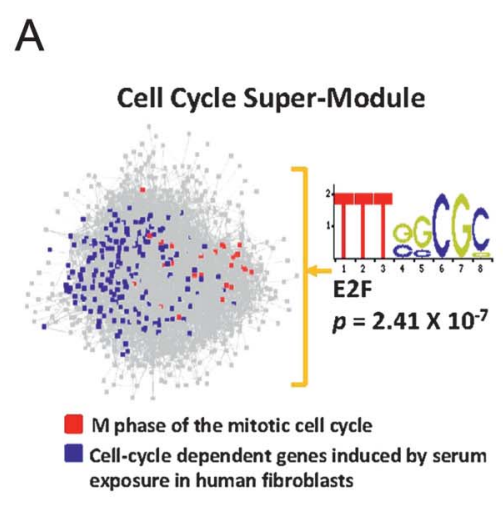

Immune Response Super-Module

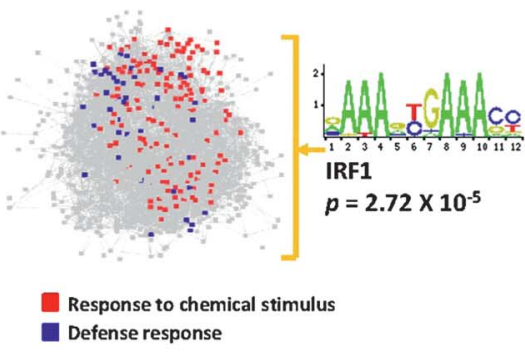

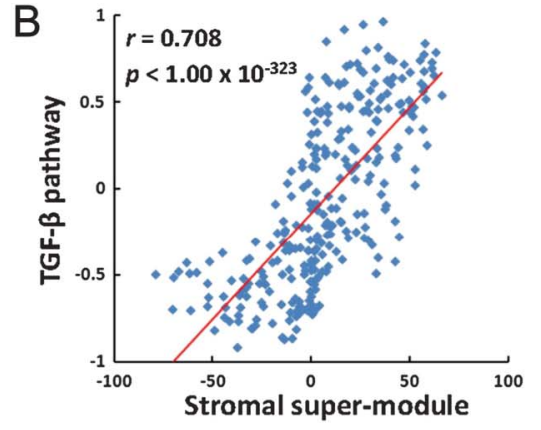

C

Stromal Super-Module

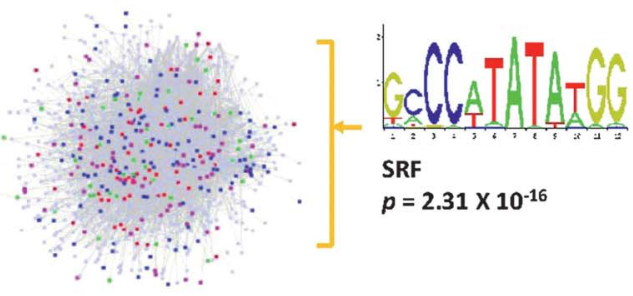

ECM
Cell surface receptor linked signal transduction
Establishment of localisation
Membrane

Figure 3 Oncogenic pathways and module expression. (A) Transcription factor-binding motifs in super-modules. Network diagrams depict the cell cycle and immune super-modules. Blue and red points represent distinct modules within the larger super-module. E2F consensus binding sites were significantly enriched in genes of the cell cycle super-module $\left(p=2.41 \times 10^{-7}\right)$, while IRF1 consensus binding sites were significantly enriched in genes of the immune super-module $\left(p=2.72 \times 10^{-5}\right)$. Position-weight matrix depictions of E2F1- and IRF1-binding motifs are shown. (B) Correlation of TGF- $\beta$ pathway with stromal super-module expression in the $288 \mathrm{GCs}$ (correlation coefficient $r=0.708, p<1.0 \times 10^{-323}$ ). The y axis represents levels of TGF- $\beta$ pathway activation, while the $x$ axis represents levels of stromal super-module expression. Each point depicts an independent sample. (C) Enrichment of serum response factor transcription factor-binding motifs in the stromal super-module $\left(p=2.31 \times 10^{-16}\right)$. Green, pink and red points in the network diagram represent distinct modules within the super-module. 
modules related to immune function $(\mathrm{r}=0.510, \mathrm{p}<0.001$; figure $2 \mathrm{~B}$, right panel). A promoter analysis of genes from the immune modules revealed that they were characterised by an enrichment of NF-kB target genes $\left(p=1.51 \times 10^{-6}\right.$, hypergeometric test $)$ and genes with interferon regulatory factor 1 (IRF1)-binding motifs $\left(p=2.72 \times 10^{-5}\right.$; figure $\left.3 \mathrm{~A}\right)$. IRF1 has been described as a major downstream target of both TNF and NF-kB signalling. ${ }^{32}$

Expression of the stromal super-module was associated with elevated levels of TGF- $\beta$ pathway activation in both, the combined validation series and the individual datasets $(r=0.708$, $\mathrm{p}<1.0 \times 10^{-323}$; figure $3 \mathrm{~B}$ and online supplemental figure 4). Moreover, expression of the TGF- $\beta$ ligands TGF- $\beta 3$ and TGF- $\beta 1$, and also the TGF- $\beta$ receptors TGF- $\beta$ R1, TGF- $\beta$ R 2 and TGF- $\beta$ R 3 was consistently higher in GCs with high stromal super-module expression (all p values $<0.001$; online supplemental table 10). A promoter analysis of genes in the stromal super-module revealed that they were significantly enriched in genes with serum response factor-binding motifs $\left(\mathrm{p}=2.31 \times 10^{-16}\right.$; figure $\left.3 \mathrm{C}\right)$, a transcription factor known to be activated by TGF- $\beta$ signalling. ${ }^{33-35}$ These results suggest that TGF- $\beta$ signalling may regulate the expression of the stromal super-module in GC.

Similar to the clinicopathological characteristics, associations between the expression modules and oncogenic pathways observed in the combined validation series were largely preserved when the three independent validation datasets (SG-3, AU-2 and YGC) were analysed individually (online supplemental table 9).

\section{Expression of the stromal super-module in GCs predicts overall survival}

Surveying the different expression modules, we identified a significant relationship between the expression level of the stromal super-module in GC and patient survival. Specifically, in the combined validation dataset, univariate Cox regression analysis treating stromal super-module expression as a continuous variable in the model revealed that GCs with a high level of stromal super-module expression were associated with significantly poorer overall survival $(\mathrm{p}=0.025 ; H R 1.007,95 \%$ CI 1.001 to 1.013 ; table 2). Similar results were observed in a KaplanMeier survival analysis, comparing the $1 / 3$ of patients with high stromal super-module expression with the $1 / 3$ of patients with low stromal super-module expression $(p=0.004$, log-rank test; figure 4). Adverse survival trends of patients with GCs exhibiting high stromal super-module expression were also observed when the individual datasets were analysed ( $\mathrm{p}=0.043$ (SG-3); $\mathrm{p}=0.045$ (YGC); $\mathrm{p}=0.23$ (AU-2); online supplemental figure 5). Further supporting the association between patient survival and stromal super-module expression, a significant relationship with patient survival was also observed in a fourth dataset (AMS, $\mathrm{n}=34$; comparing top vs bottom tertiles), which was used in the initial construction of the coexpression data, and for which clinical outcome data was available $(p=0.018$; online supplemental figure 5).

To compare the prognostic significance of stromal supermodule expression with current gold standard clinicopathological criteria of patient prognosis prediction, we performed multivariate Cox regression analysis incorporating stromal super-module expression (continuous variable), age ( $<65$ years vs $\geq 65$ years), gender, resection margin status (R0 vs R1), morphology (intestinal-type vs diffuse-type vs mixed type, grade of differentiation (well vs moderate vs poor) and pathological tumour stage according to TNM classification (UICC/AJCC 6th edition). In the combined validation series, the prognostic value

Table 2 Univariate and multivariate Cox regression analysis for stromal super-module expression and clinicopathological characteristics in the combined validation series

\begin{tabular}{|c|c|c|c|c|}
\hline \multirow[b]{2}{*}{ Covariate } & \multicolumn{2}{|l|}{ Univariate } & \multicolumn{2}{|l|}{ Multivariate } \\
\hline & HR $(95.0 \% \mathrm{Cl})$ & $p$ Value & HR $(95.0 \% \mathrm{Cl})$ & p Value \\
\hline \multicolumn{5}{|c|}{ Stromal module expression } \\
\hline Continuous variable & $1.007(1.001$ to 1.013$)$ & 0.025 & 1.005 (0.999 to 1.012$)$ & 0.12 \\
\hline \multicolumn{5}{|l|}{ Age } \\
\hline$<65$ years & 1 & & 1 & \\
\hline$\geq 65$ years & 1.501 (1.072 to 2.103$)$ & 0.018 & 1.690 (1.169 to 2.442 ) & 0.005 \\
\hline \multicolumn{5}{|l|}{ Gender } \\
\hline Female & 1 & & 1 & \\
\hline Male & $1.488(1.035$ to 2.141$)$ & 0.032 & 1.287 (0.858 to 1.931$)$ & 0.223 \\
\hline \multicolumn{5}{|l|}{ Morphology } \\
\hline Intestinal & 1 & & - & - \\
\hline Diffuse & $1.167(0.819$ to 1.661$)$ & 0.392 & - & - \\
\hline Unclassifiable & $1.002(0.584$ to 1.718$)$ & 0.995 & - & - \\
\hline \multicolumn{5}{|l|}{ Resection margin status } \\
\hline Ro & 1 & & - & - \\
\hline $\mathrm{R}$ & 1.511 (0.89 to 2.564$)$ & 0.126 & - & - \\
\hline \multicolumn{5}{|l|}{ Grade of differentiation } \\
\hline G1 & 1 & & 1 & \\
\hline $\mathrm{G} 2$ & 4.242 (1.525 to 11.801$)$ & 0.006 & $2.926(1.029$ to 8.320$)$ & 0.044 \\
\hline G3 & 4.735 (1.731 to 12.949$)$ & 0.002 & 3.429 (1.229 to 9.569$)$ & 0.019 \\
\hline \multicolumn{5}{|l|}{ AJCC staging } \\
\hline Stage I & 1 & & 1 & \\
\hline Stage II & $2.346(1.045$ to 5.264$)$ & 0.039 & 1.924 (0.853 to 4.341$)$ & 0.115 \\
\hline Stage III & 4.649 (2.308 to 9.366$)$ & $<0.001$ & $4.126(2.023$ to 8.415$)$ & $<0.001$ \\
\hline Stage IV & 12.002 (5.801 to 24.833$)$ & $<0.001$ & 10.786 (5.035 to 23.106$)$ & $<0.001$ \\
\hline
\end{tabular}


A

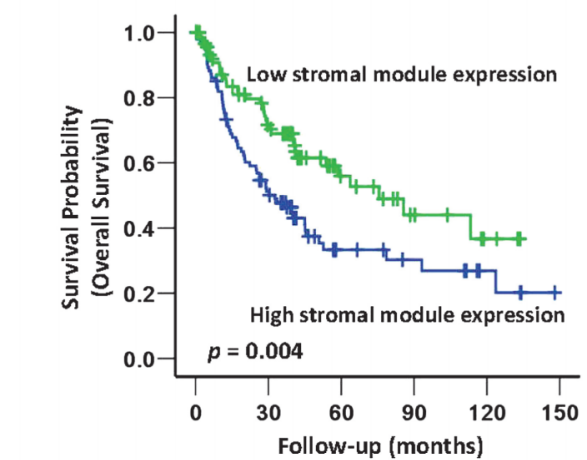

Patients at risk

Low stromal: $\quad \begin{array}{lllllll}95 & 53 & 17 & 8 & 3 & 0\end{array}$

B

Patients with diffuse-type GC

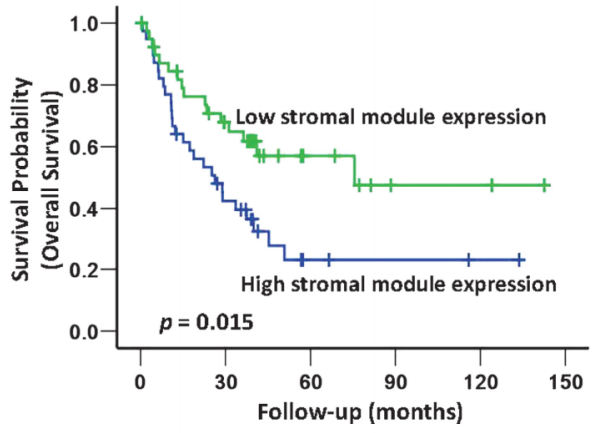

Patients at risk

$\begin{array}{lllllll}\text { Low stromal: } & 40 & 22 & 7 & 2 & 2 & 0 \\ \text { High stromal: } & 40 & 15 & 3 & 2 & 1 & 0\end{array}$

Figure 4 Stromal super-module expression predicts patient survival in the cancers from the combined validation dataset, and in patients with diffuse-type gastric cancer (GC). (A) Expression of the stromal super-module predicts all GC patient survivals in the combined validation dataset. All GC patients were divided into three equally sized groups based on the level of stromal super-module expression: high stroma (top 1/3), moderate stroma (middle 1/3), and low stroma (bottom 1/3). Kaplan-Meier analysis was used to compare overall survival probability from the time of surgery of patients with either high stroma (top 1/3) or low stroma (bottom 1/3) GC. GC patients with high levels of stromal super-module expression had a poorer prognosis ( $p=0.004$, log-rank test). (B) Stromal super-module expression levels predict patient survival in patients with diffuse-type GC. One hundred and eighteen patients with diffuse-type GC (combined SG-3/AU-2/YGC data) were divided into three equally sized groups based on levels of stromal super-module expression: high stroma (top 1/3), moderate stroma (middle 1/3), and low stroma (bottom 1/3). Kaplan-Meier survival analysis demonstrates that patients with diffuse-type GC and high levels of stromal super-module expression (top $1 / 3, n=40$ ) had poorer survival than patients with diffuse-type GC and low levels of stromal super-module expression (bottom $1 / 3, n=40)(p=0.015$, log-rank test).

of stromal super-module expression was related to disease stage ( $p=0.12$, HR: $1.005,95 \%$ CI 0.999 to 1.012 ; table 2 ). A stageadjusted analysis revealed that the association between stromal super-module expression and survival was primarily observed in stages III and IV (ie, late stage) disease $(p=0.024$ for stages III and IV compared with $\mathrm{p}=0.668$ for stages I and II; online supplemental table 11). Kaplan-Meier survival analysis restricted to stage III GC patients showed that patients with GCs expressing high levels of stromal super-module expression had a poorer prognosis compared with patients with low stromal supermodule expression ( $p=0.039$; online supplemental figure 6$)$.

Besides survival, high expression of the stromal super-module was significantly associated with diffuse-type morphology $\left(\mathrm{p}<1.00 \times 10^{-323}\right.$; online supplemental table 6$)$, a histological subtype traditionally associated with clinically poor prognosis. ${ }^{36}$ In a subgroup analysis of diffuse-type GCs in the combined validation set, we found that patients with diffuse-type and high stromal super-module expressing GCs exhibited a significantly poorer survival compared with patients with diffusetype and low stromal super-module expressing GCs $(p=0.015$; figure 4B). This result suggests that additional information beyond histological classification may be revealed by measuring stromal super-module expression, supporting recent reports describing distinct subtypes of diffuse-type GC. ${ }^{38}$

To validate our results using a non-array technology, we performed immunohistochemistry on GC full sections for two proteins, vimentin (VIM) and caldesmon (CALDESM), whose mRNA expression was highly correlated with stromal supermodule expression (see Methods for selection criteria). In the LS-1 dataset, VIM protein expression exhibited a trend towards association with stromal super-module expression $(\mathrm{r}=0.34$, $\mathrm{p}=0.08$ ). Similarly, CALDESM protein expression tended to be positively associated with stromal super-module expression $(r=0.22, p=0.26$; online supplemental figure 7$)$. We think it not surprising that there is only a trend and no significant correlation between the IHC results and the stromal super-module expression, as the former is based on measurements of a single biomarker (eg, VIM or CALDESM protein), while the latter is based upon the coordinated expression levels of 878 genes, which is likely to be more robust.

\section{Stromal super-module expression is related to the intratumoural stroma proportion}

We hypothesised that GC stromal super-module expression levels might be correlated with specific histopathological features observable in routine haematoxylin \& eosin (H\&E)-stained sections of GCs. To explore this possibility, we analysed two GC datasets, representing cases for which we were able to obtain full H\&E sections of sufficient quality for histological analysis, and corresponding gene expression data. These included (1) 156 GCs comprising 119 SG-3 GCs, and an additional 37 GCs that were subsequently recruited in the course of this study ('Expanded SG-3') and (2) all 29 GCs in the LS-1 dataset. Genomic and histological analysis confirmed that the GCs exhibited highly variable stromal super-module expression across individual GCs (figure 5A for LS-1). A representative H\&E-stained section was selected from each case and scanned using an Aperio scanner. To quantify the proportions of the different components within the cancer (eg, cancer cells, stroma including fibroblasts and extracellular matrix, tumour lumen, necrosis, vessels, inflammatory cells), we used a computerised morphometric method (point counting, see online supplemental methods), previously applied to colon cancer ${ }^{39}$ and formally described by Weibel. ${ }^{40}$ The set of investigated GCs exhibited diverse histopathological phenotypes with respect to tumour cell density, intra-tumoural stroma, vascularity and immune cell infiltrates (figure $5 \mathrm{~B}$ and $\mathrm{C}$ ). We detected a significant positive correlation between the expression of the stromal super-module and the morphometrically quantified proportion of intratumoural stroma in both, the expanded SG-3 and LS-1 datasets (SG-3: median ITS: 60\%, range: 15-99\%, correlation coefficient $\mathrm{r}=0.327, \mathrm{p}=3.14 \times 10^{-5}$, figure 5D; LS-1: $29 \mathrm{GCs}$, median ITS: 47\%, range: 3-88\%; correlation coefficient $\mathrm{r}=0.426, \mathrm{p}=0.021$; online supplemental figure 8 ). The 

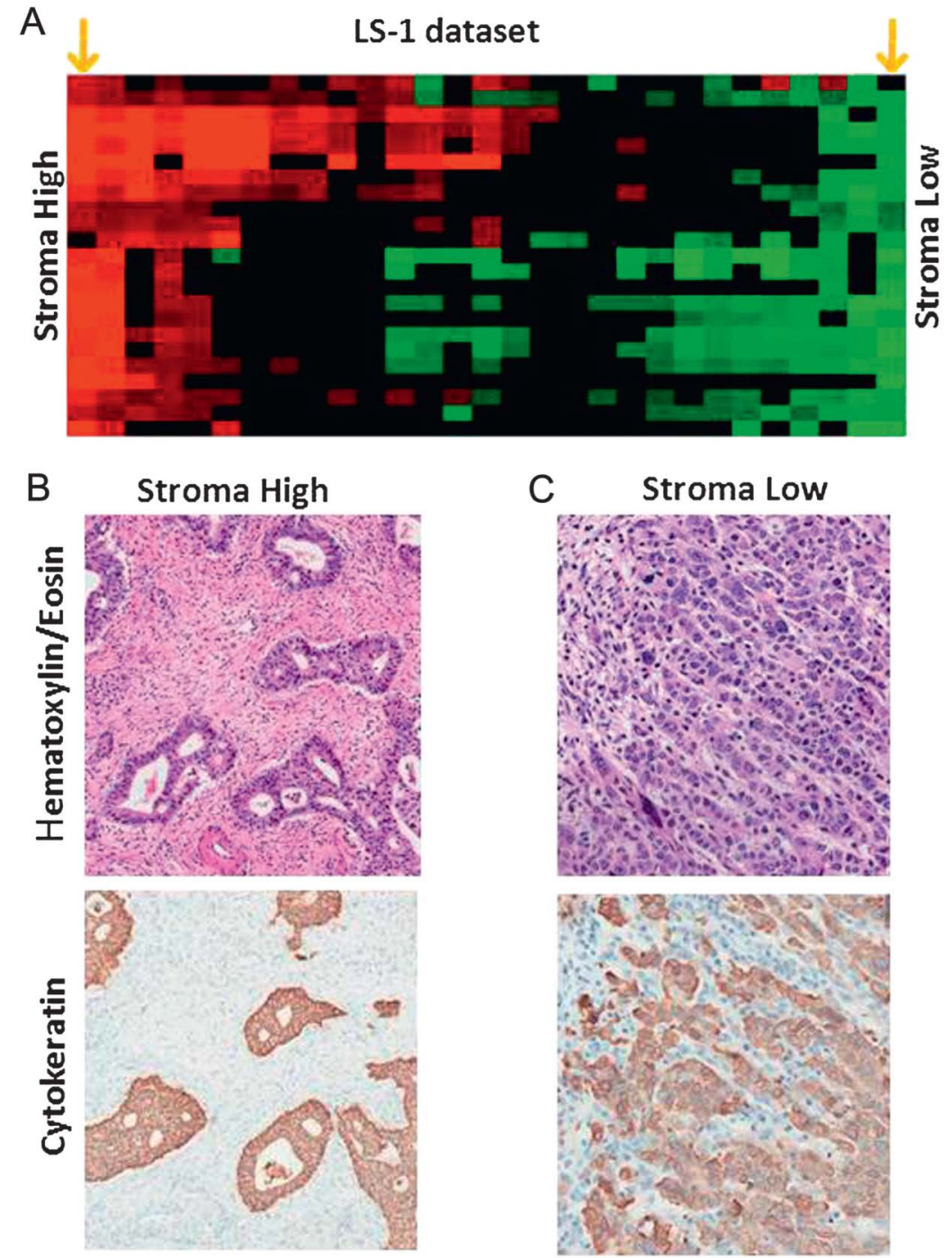
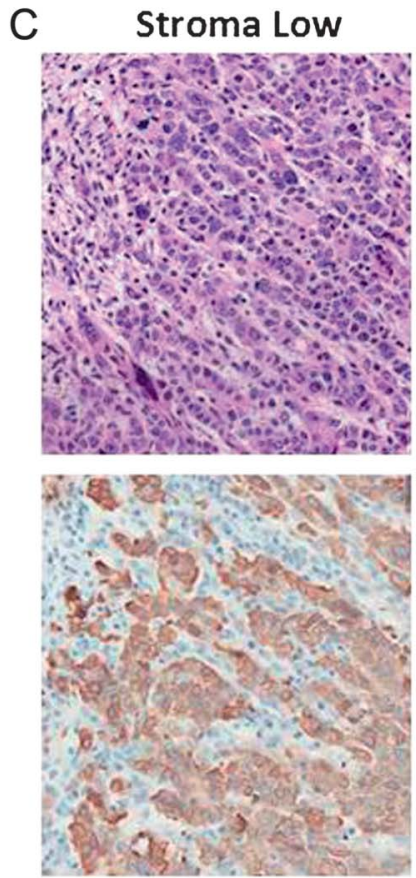
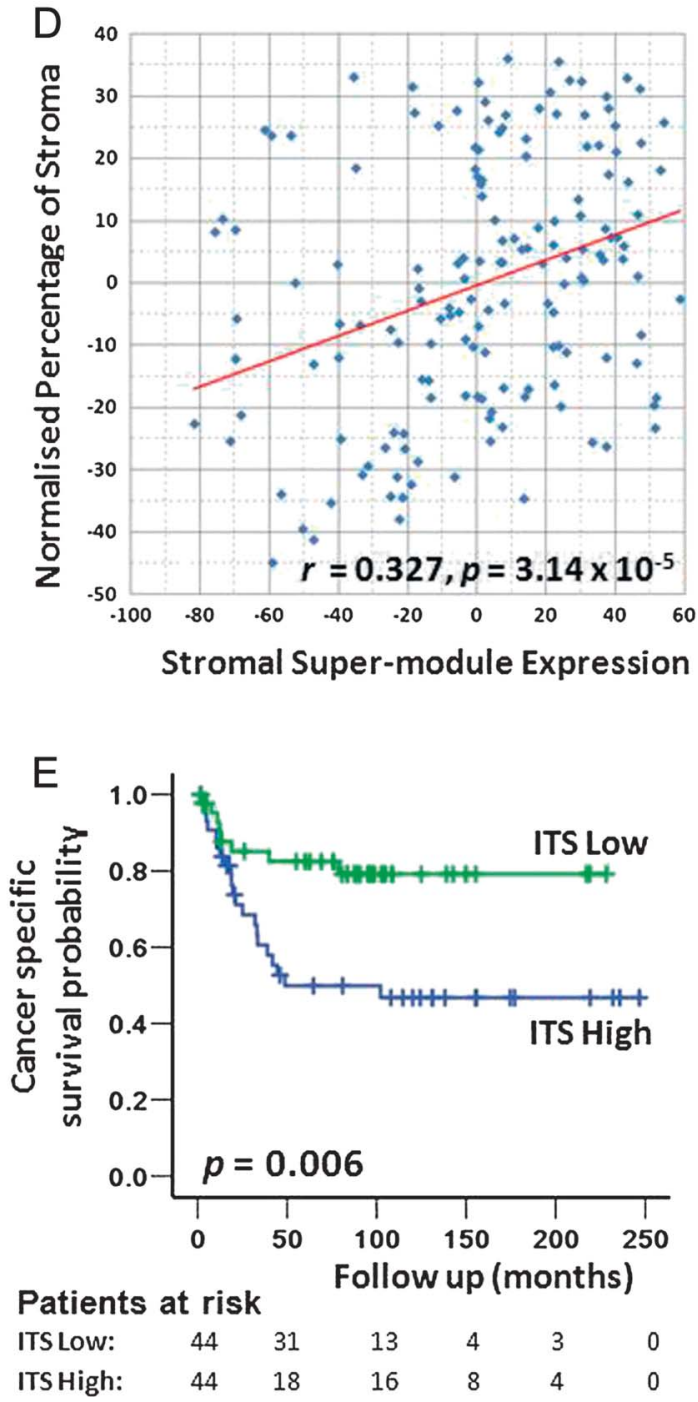

Figure 5 Association of intra-tumoural stroma (ITS) proportion with stromal super-module expression and patient survival. (A) Stromal super-module expression of gastric cancers (GCs) in the LS-1 dataset $(n=29)$. Columns represent individual GCs, rows represent individual stromal modules. Red, high stromal super-module expression, green, low stromal super-module expression. The orange arrows at the extreme left and right represent GCs selected for figure 5B and C. (B) and (C) Representative H\&E-stained sections of GCs (top panel) illustrating prominent differences in ITS between a GC with high stromal super-module expression (B) and a GC with low stromal super-module expression (C). Consecutive sections from the same blocks subjected to cytokeratin (CK) immunohistochemistry to facilitate visualisation of GC tumour cells (brown). The intra-tumoural stroma was CK negative (blue due to the haematoxylin counterstain). (D) Association of stromal super-module expression with the ITS proportion in the expanded SG-3 dataset. The y axis represents the normalised ITS proportion measured by morphometry (see Methods). The $x$ axis represents the expression level of the stromal super-module. Each point represents an individual GC. A significant positive correlation was observed (correlation coefficient $r=0.327, p=3.14 \times 10^{-5}$ ). (E) $131 \mathrm{GCs}$ from LS-2 were divided into three equal-sized groups based on the levels of ITS, for example GCs with high, moderate or low ITS proportion. Kaplan-Meier analysis demonstrates that patients with GCs exhibiting a high ITS proportion (blue line) have poorer cancer-specific survival compared to patients with low ITS proportion GCs (green line, p=0.006, log-rank test).

association between stromal super-module expression and ITS proportion remained significant after correcting for stage, age, gender, Laurén classification and grade (expanded SG-3: $\mathrm{p}=0.002$; LS $-1: \mathrm{p}=0.024$; partial correlation analysis ${ }^{41}$ ). This result suggests that the expression level of the stromal supermodule in GCs may be directly related to the proportion of ITS measured in H\&E-stained tissue sections.

To further investigate the stromal super-module expression/ ITS proportion association, we tested whether the ITS proportion directly measured from H\&E-stained tissue samples, might predict GC patient survival. Applying the same computerised point counting method, we analysed another independent series of 131 GC patients from which TMAs had been constructed (LS-2, see Methods, online supplemental table 2 provides the clinicopathological characteristics of the TMA cohort). A high ITS proportion was more commonly seen in diffuse-type GC than intestinal-type GC (median ITS diffuse-type GC: 64\%, range: 17-95\%; median ITS intestinal-type GC: 47\%, range: $11-92 \% ; \mathrm{p}=0.005$; Student's $\mathrm{t}$ test), in late-stage GCs than early-stage GCs (median ITS late-stage GCs: 62\%, range: 1195\%; median ITS early-stage GCs: 47\%, range: 13-92\%; $\mathrm{p}=0.01$ ), and in high-grade GCs than low-grade GCs (median ITS high-grade GCs: 57\%, range: 13-95\%; median ITS lowgrade GCs: $47 \%$, range: $11-82 \% ; \mathrm{p}=0.007)$. Similar to the genomic results, a Kaplan-Meier survival analysis comparing the one third of patients with high ITS GCs with one third of patients with low ITS GCs revealed that patients with high ITS GC had a significantly poorer prognosis $(p=0.006$, log-rank 
test; figure 5E). A trend towards worsened survival was also seen in ITS high diffuse-type GCs $(p=0.29$, log-rank test). However, in multivariate analysis, including ITS proportion (continuous variable), age ( $<65$ years vs $\geq 65$ years), gender (male vs female), morphology (intestinal-type vs diffuse-type vs mixed type), grade of differentiation (well vs moderate vs poor) and pathological tumour stage according to TNM classification (UICC/AJCC 6th edition) in the Cox regression model, ITS proportion was not an independent predictor of patient survival $(\mathrm{p}=0.682$; online supplemental table 12$)$.

Similar to the LS-2 dataset, in the expanded SG-3 dataset, a high ITS proportion was more commonly seen in diffuse-type GCs than intestinal-type GC (median ITS diffuse-type GC: 67\%, range: 15-96\%; median ITS intestinal-type GC: 53\%, range: $18-92 \% ; \mathrm{p}=9.204 \times 10^{-5}$ ), in high-grade GCs than lowgrade GCs (median ITS high grade GCs: 64\%, range: 15-96\%; median ITS low-grade GCs: 55\%, range: 20-92\%; $\mathrm{p}=0.044)$, and tended to be more common in late-stage GCs than earlystage GCs (median ITS late-stage GCs: 63\%, range: 18-95\%; median ITS early-stage GCs: 53\%, range: $15-96 \%$; $\mathrm{p}=0.076$ ). Most importantly, when divided into three equal-sized groups based on their ITS proportions, SG-3 patients with high ITS GCs also exhibited a poorer overall survival compared with patients with low ITS GCs $(p=0.047$; online supplemental figure 9). The similarity of these relationships between clinicopathological data, patient survival and morphometry results compared with the genomic analysis results, both derived from entirely independent GC datasets, supports the potential existence of a biological relationship between the level of stromal super-module expression and the ITS proportion in GCs.

\section{DISCUSSION}

This study reports the largest genomic meta-analysis of GC to date exceeding previous studies by more than double the total number of patients. ${ }^{22}$ Compared with previous GC gene expression studies from single centres, ${ }^{15-20}$ our meta-analysis of multiple expression datasets provided increased statistical power enabling us to identify significant gene-gene relationships which would most likely be less evident in smaller datasets. ${ }^{22}$ For example, demonstrating the increased sensitivity of the current study, we were able to detect the expression of several modules related to mitochondrial and proteosomal function, which were not evident in our own previous analyses of smaller subsets of the same data. ${ }^{15-20}$ Previous studies based on data from a single microarray platform may also suffer from biases due to platform-specific artifacts. ${ }^{42}$ In contrast, our meta-analysis approach combined data from multiple independent patient populations and different microarray platforms, maximising the probability that identified relationships are biologically relevant.

Our analysis indicates that the GC coexpression network exhibits a 'scale-free' topological organisation where the vast majority of genes are connected to a small number of other genes ('edges'), and only a few genes ('hubs') are highly connected to many other genes. ${ }^{43-45}$ A survey of the top 'hub' genes revealed involvement in normal gastric physiology (PGC, $L I P F)$, cell adhesion (NCAM1, LGALS4, MGP), gene transcription (ILF2, H2AFZ), and signalling (PPP2R3A, PPTRC) (online supplemental table 5 ). It is possible that these 'hub' genes may function as important control nodes in the GC gene coexpression networks, however, without direct functional data the functional significance of the 'hub' genes in the GC coexpression network remains to be elucidated. In addition to hub genes, our analysis revealed the existence of 178 expression modules associated with diverse cellular functions ranging from cell proliferation and immunity to mitochondrial and stromal function. Some of the identified expression modules may highlight therapeutic opportunities. For example, we observed high expression of modules related to proteasomal function in a subset of GCs. Bortezomib, a clinically approved proteasome inhibitor, has recently shown pre-clinical efficacy in GC cells. ${ }^{46}$ It may be interesting to investigate if GCs expressing high levels of the proteasomal modules exhibit heightened sensitivity to bortezomib or other related proteasomal inhibitor compounds. We also identified relationships between modules, for example, GCs with high expression of cell proliferation modules tended to coexpress modules related to digestive function, while GCs with high expression of the stromal super-module exhibited low levels of proliferation and digestive module expression. The mutual exclusivity of the expression of the stroma and proliferation modules observed in this study is consistent with recent studies demonstrating that cancer cells can exist in either a proliferative or invasive state, but usually cannot proliferate and invade at the same time. ${ }^{47-49}$

We found that the expression level of a TGF- $\beta$ associated super-module of stroma-related genes consistently predicted clinical outcome in multiple independent GC datasets. Expression of the stromal super-module was also related to the ITS proportion, a specific morphological GC phenotype and diffuse-type histology. These genomic and morphometric results are of clinical relevance, as they suggest that the prognosis of GC patients may be influenced by cancer cells and by other cell types residing in the tumour stroma. Our results also provide a molecular basis that may explain, at least in part, the poor prognosis of diffuse-type GC patients. First, the negative correlation between stroma and cell proliferation may contribute to the poor response of diffuse-type GCs to cytotoxic chemotherapy, which targets actively dividing cells. Second, a high ITS proportion may directly inhibit the effects of current therapies by reducing both drug delivery to tumour cells ${ }^{5051}$ and protecting cells against chemotherapy-induced apoptosis. ${ }^{52}$ Third, recent functional studies have indicated that the tumour stroma may play a vital role in the differentiation, proliferation and migration of tumour cells, ${ }^{53}$ and the tumor stroma may contribute to aggressive disease by providing a favourable mechano-environmental scaffold necessary for tumour progression. ${ }^{54}$ These findings are supported by studies in different cancer types including oesophageal, ${ }^{55}$ colorectal, ${ }^{39} 56$ prostate, ${ }^{57}$ pancreatic, ${ }^{58}$ breast and liver cancers, ${ }^{59}$ which showed that the tumour microenvironment and stroma may play significant roles in patient prognosis and chemosensitivity. ${ }^{60-63}$ However, to our knowledge, our report is the first to demonstrate a prognostic role of the tumour stroma in GC, highlighting the potential role of the ITS proportion as a predictive biomarker to identify subgroups of patients with GCs that might respond to therapies directed towards the tumour stroma.

Given the association between stromal super-module expression levels and GC patient survival, and the current limited impart of targeted agents (trastuzumab, lapatinib) in diffuse-type GCs, we were interested in identifying the molecular pathways potentially influencing intra-tumoural stroma growth. By correlating the expression levels of the stromal super-module to the activity of different oncogenic-signalling pathways, our analysis strongly implicates the TGF- $\beta$ signalling pathway as a key regulator of the intra-tumoural stroma. Although the TGF- $\beta$ pathway has been historically viewed as a tumour-suppressive pathway where tumour cells often exhibit mutational or epigenetic inactivation of TGF- $\beta$ pathway components, such as TGFBRI, TGF $\beta R I I$ and SMAD4, ${ }^{64}$ recently published work 
suggests that TGF- $\beta$ signalling in tumours is more complex and may stimulate a pro-tumourigenic stromal environment. ${ }^{65}$ For example, TGF- $\beta$ ligands secreted by cancer cells have been shown to alter the function of healthy fibroblasts within the tumour stroma, leading to a myofibroblast-like phenotype supporting tumour growth, vascularisation and metastasis. ${ }^{66} 67$ Notably, the TGF- $\beta$ pathway has been identified as a target for therapeutic intervention using endogenous proteins, such as soluble betaglycan or decorin, or artificial agents, such as antisense oligonucleotides, antibodies or small-drug molecules. ${ }^{64}$ Given the dearth of therapeutic options for GC patients at present, it will be important to assess if targeting TGF- $\beta$ might prove an effective strategy for perturbing the GC tumour stroma and improving patient outcomes.

In summary, this is the first comprehensive genomic meta-analysis of GC transcriptome data, generating a robust inventory of multiple gene-expression modules present in GCs. Our analysis revealed that the level of stromal super-module expression in GCs may serve as a novel prognostic factor in GC, and that this pathway is likely to involve TGF- $\beta$ signalling. Admittedly, the association between patient survival and stromal gene expression/ITS proportion, while statistically significant, is relatively weak with regard to effect size. As such, it remains currently uncertain whether measuring the ITS proportion will prove to be a useful clinical tool for predicting GC patient prognosis, above and beyond the accepted standard of TNM tumour staging. To definitively address this question, future research goals will involve measuring the ITS proportion in patient materials from large prospective multicentre randomised controlled trial populations, where potential biases, due to disease stage, patient-related factors, treatment, pathology reporting and tissue collection are minimised. Finally, very few of our patients received chemotherapy prior to surgery, and hence, our results cannot address the prognostic value of the ITS proprotion after neoadjuvant chemotherapy. Given the increasing use of neoadjuvant chemotherapy in GC patients in the West, ${ }^{68} 69$ it will be intriguing to evaluate the impact of neo-adjuvant chemotherapy on ITS at the histological and molecular level.

\footnotetext{
Author affiliations

${ }^{1}$ Cellular and Molecular Research, National Cancer Centre, Singapore

${ }^{2}$ Pathology and Tumour Biology, Leeds Institute of Molecular Medicine, University of Leeds, UK

${ }^{3}$ Division of Medical Oncology, National Cancer Centre, Singapore

${ }^{4}$ Cancer and Stem Cell Biology, Duke-NUS Graduate Medical School, Singapore

${ }^{5}$ Department of Medicine, Yong Loo Lin School of Medicine, National University of

Singapore

${ }^{6}$ Department of Pathology, Free University Medical Center Amsterdam, The Netherlands

${ }^{7}$ Department of Internal Medicine, Yonsei Cancer Centre, South Korea

${ }^{8}$ Departments of Gastrointestinal Medical Oncology, MD Anderson Cancer Centre, USA

${ }^{9}$ Department of Surgery, Yonsei University College of Medicine, South Korea

${ }^{10}$ Department of Pathology, Singapore General Hospital, Singapore

${ }^{11}$ Cancer Genomics and Biochemistry Laboratory, Peter MacCallum Cancer Centre,

East Melbourne, Victoria, Australia

${ }^{12}$ Department of Medicine (RMH/WH), University of Melbourne, Western Hospital, Footscray, Victoria, Australia

${ }^{13}$ Systems Biology, Division of Cancer Medicine, MD Anderson Cancer Centre, USA

${ }^{14}$ Cancer Science Institute of Singapore, Yong Loo Lin School of Medicine, National University of Singapore

${ }^{15}$ Genome Institute of Singapore, Singapore
}

Acknowledgements The authors thank Ken Hillan from Genentech for supporting the generation of the Leeds microarray dataset.

Contributors YW and HG contributed equally to this work. YW, HG and PT conceived and designed the experiments. HG, TI, JM AW, LKH, WN and HG performed the experiments. YW, CHO, JL and HG analysed the data. HG, IBT, JW,
ML, JHK, KGY, NvG, BY, SYR, JAA, JHC, SHN, LKH, AB and J-SL contributed reagents/materials/analysis tools. YW, HG and PT wrote the paper.

Funding This work was supported by Grants to PT from the Biomedical Research Council of Singapore (Grant 05/1/31/19/423), the National Medical Research Council of Singapore (Grant TCR/001/2007), and internal grants from the Duke-National University of Singapore Graduate Medical School, and the Cancer Sciences Institute of Singapore. The funders had no role in study design, data collection and analysis, decision to publish or preparation of the manuscript.

Competing interests None.

Patient consent Obtained.

Ethics approval Primary gastric cancer tissue samples were collected with patient consent from the participating centre's tissue repositories or pathology archives and approval by the respective institutional Research Ethics Review Committees in accordance with local regulations and legislations. Clinical information was collected with Institutional Review Board approval.

Provenance and peer review Not commissioned; externally peer reviewed.

Data sharing statement The microarray data in this study is available from GEO under accession numbers GSE2669, GSE2680, GSE2685, GSE2637, GSE3438, GSE15459, GSE13861, GSE37023, GSE35809 and http://smd.stanford.edu/cgi-bin/ publication/viewPublication.pl?pub_no $=516$.

\section{REFERENCES}

1 Parkin DM, Bray F, Ferlay J, et al. Global cancer statistics, 2002. CA Cancer J Clin 2005;55:74-108.

2 Hartgrink $\mathrm{HH}$, Jansen EP, van Grieken NC, et al. Gastric cancer. Lancet 2009:374:477-90.

3 Jackson C, Cunningham D, Oliveira J. Gastric cancer: ESMO clinical recommendations for diagnosis, treatment and follow-up. Ann Oncol 2009;20(Suppl 4):34-6.

4 Ahn JR, Jung M, Kim C, et al. Prognosis of pN3 stage gastric cancer. Cancer Res Treat 2009:41:73-9.

5 Grabsch H, Kerr D, Quirke P. Is there a case for routine clinical application of ploidy measurements in gastrointestinal tumours? Histopathology 2004:45:312-34.

6 Grabsch H, Sivakumar S, Gray S, et al. HER2 expression in gastric cancer: rare, heterogeneous and of no prognostic value-conclusions from 924 cases of two independent series. Cell Oncol 2010;32:57-65.

7 Dicken BJ, Bigam DL, Cass C, et al. Gastric adenocarcinoma: review and considerations for future directions. Ann Surg 2005;241:27-39.

8 Nobili S, Bruno L, Landini I, et al. Genomic and genetic alterations influence the progression of gastric cancer. World J Gastroenterol 2011;17:290-9.

9 Scartozzi M, Galizia E, Freddari F, et al. Molecular biology of sporadic gastric cancer: prognostic indicators and novel therapeutic approaches. Cancer Treat Rev 2004;30:451-9

10 Haas M, Dimmler A, Hohenberger W, et al. Stromal regulatory T-cells are associated with a favourable prognosis in gastric cancer of the cardia. BMC Gastroenterol 2009;9:65.

11 Ishigami S, Natsugoe S, Tokuda K, et al. Prognostic value of intratumoral natural killer cells in gastric carcinoma. Cancer 2000;88:577-83.

12 Caruso RA, Bellocco R, Pagano $M$, et al. Prognostic value of intratumoral neutrophils in advanced gastric carcinoma in a high-risk area in northern Italy. Mod Pathol 2002;15:831-7.

13 Lee HE, Chae SW, Lee YJ, et al. Prognostic implications of type and density of tumour-infiltrating lymphocytes in gastric cancer. Br J Cancer 2008;99:1704-11.

14 Alvarado MD, Jensen EH, Yeatman TJ. The potential role of gene expression in the management of primary and metastatic colorectal cancer. Cancer Control 2006; 13:27-31

15 Tsai CJ, Herrera-Goepfert R, Tibshirani RJ, et al. Changes of gene expression in gastric preneoplasia following Helicobacter pylori eradication therapy. Cancer Epidemiol Biomark Prev 2006;15:272-80.

16 Boussioutas A, Li H, Liu J, et al. Distinctive patterns of gene expression in premalignant gastric mucosa and gastric cancer. Cancer Res 2003:63:2569-77.

17 Chen X, Leung SY, Yuen ST, et al. Variation in gene expression patterns in human gastric cancers. Mol Biol Cell 2003;14:3208-15.

18 Hippo Y, Taniguchi $\mathrm{H}$, Tsutsumi $\mathrm{S}$, et al. Global gene expression analysis of gastric cancer by oligonucleotide microarrays. Cancer Res 2002;62:233-40.

19 Tay ST, Leong SH, Yu K, et al. A combined comparative genomic hybridization and expression microarray analysis of gastric cancer reveals novel molecular subtypes. Cancer Res 2003:63:3309-16.

$20 \mathrm{Kim}$ SY, Kim JH, Lee HS, et al. Meta- and gene set analysis of stomach cancer gene expression data. Mol Cells 2007:24:200-9.

21 Cho JY, Lim JY, Cheong JH, et al. Gene expression signature-based prognostic risk score in gastric cancer. Clin Cancer Res 2011;17:1850-7.

22 Aggarwal A, Guo DL, Hoshida Y, et al. Topological and functional discovery in a gene coexpression meta-network of gastric cancer. Cancer Res 2006;66:232-41. 
23 Albert R. Scale-free networks in cell biology. J Cell Sci 2005;118:4947-57.

24 Wei H, Persson S, Mehta T, et al. Transcriptional coordination of the metabolic network in Arabidopsis. Plant Physiol 2006;142:762-74.

25 Chang HY, Sneddon JB, Alizadeh AA, et al. Gene expression signature of fibroblast serum response predicts human cancer progression: similarities between tumours and wounds. PLOS Biol 2004;2:E7.

26 Segal E, Friedman N, Koller D, et al. A module map showing conditional activity of expression modules in cancer. Nat Genet 2004;36:1090-8.

27 IARC. WHO Classification Of Tumours Of The Digestive System. Lyon, France: International Agency for Research on Cancer, 2010.

28 Ooi CH, Ivanova T, Wu J, et al. Oncogenic pathway combinations predict clinical prognosis in gastric cancer. PLoS Genet 2009;5:e1000676.

29 Verrecchia F, Chu ML, Mauviel A. Identification of novel TGF-beta /Smad gene targets in dermal fibroblasts using a combined cDNA microarray/promoter transactivation approach. J Biol Chem 2001;276:17058-62.

30 Weston GC, Haviv I, Rogers PA. Microarray analysis of VEGF-responsive genes in myometrial endothelial cells. Mol Hum Reprod 2002;8:855-63.

31 Nahle Z, Polakoff J, Davuluri RV, et al. Direct coupling of the cell cycle and cell death machinery by E2F. Nat Cell Biol 2002;4:859-64.

32 Ten RM, Blank V, Le Bail O, et al. Two factors, IRF1 and KBF1/NF-kappa B, cooperate during induction of $\mathrm{MHC}$ class I gene expression by interferon alpha beta or Newcastle disease virus. C R Acad Sci 1993;316:496-501.

33 Sandbo N, Kregel $S$, Taurin $S$, et al. Critical role of serum response factor in pulmonary myofibroblast differentiation induced by TGF-beta. Am J Respir Cell Mol Biol 2009:41:332-8.

34 Chai J, Norng M, Tarnawski AS, et al. A critical role of serum response factor in myofibroblast differentiation during experimental oesophageal ulcer healing in rats. Gut 2007;56:621-30.

35 Yang $Y$, Zhe $X$, Phan SH, et al. Involvement of serum response factor isoforms in myofibroblast differentiation during bleomycin-induced lung injury. Am J Respir Cell Mol Biol 2003;29:583-90.

36 Cimerman M, Repse S, Jelenc F, et al. Comparison of Lauren's, Ming's and WHO histological classifications of gastric cancer as a prognostic factor for operated patients. Int Surg 1994;79:27-32.

37 Viste A, Eide GE, Halvorsen K, et al. The prognostic value of Lauren's histopathological classification system and $A B O$ blood groups in patients with stomach carcinoma. Eur J Surg Oncol 1986;12:135-41.

38 Chiaravalli AM, Klersy C, Tava F, et al. Lower- and higher-grade subtypes of diffuse gastric cancer. Hum Pathol 2009;40:1591-9.

39 West NP, Dattani M, McShane P, et al. The proportion of tumour cells is an independent predictor for survival in colorectal cancer patients. Br J Cancer 2010;102:1519-23.

40 Weibel ER. Stereological Methods. London, New York: Academic Press, 1979.

41 Australian \& New Zealand Journal Of Statistics. Oxford: Blackwell Publishers, 1998.

42 Choi JK, Yu U, Yoo OJ, et al. Differential coexpression analysis using microarray data and its application to human cancer. Bioinformatics 2005;21:4348-55.

43 van Noort V, Snel B, Huynen MA. The yeast coexpression network has a small-world, scale-free architecture and can be explained by a simple model. EMBO Rep 2004;5:280-4.

44 Jordan IK, Marino-Ramirez L, Wolf YI, et al. Conservation and coevolution in the scale-free human gene coexpression network. Mol Biol Evol 2004;21:2058-70.

45 Nayak RR, Kearns M, Spielman RS, et al. Coexpression network based on natural variation in human gene expression reveals gene interactions and functions. Genome Res 2009;19:1953-62.

46 Nakata W, Hayakawa Y, Nakagawa $H$, et al. Anti-tumor activity of the proteasome inhibitor bortezomib in gastric cancer. Int J Oncol 2011:39:1529-36.

47 Gao CF, Xie Q, Su YL, et al. Proliferation and invasion: plasticity in tumour cells. Proc Natl Acad Sci U S A 2005;102:10528-33.
48 Lee $\mathrm{HE}$, Kim MA, Lee $\mathrm{BL}$, et al. Low Ki-67 proliferation index is an indicator of poor prognosis in gastric cancer. J Surg Oncol 2010;102:201-6.

49 Anatskaya OV, Vinogradov AE. Genome multiplication as adaptation to tissue survival: evidence from gene expression in mammalian heart and liver. Genomics 2007:89:70-80.

50 Pietras K, Ostman A, Sjoquist M, et al. Inhibition of platelet-derived growth factor receptors reduces interstitial hypertension and increases transcapillary transport in tumors. Cancer Res 2001;61:2929-34.

51 Pietras K, Rubin K, Sjoblom T, et al. Inhibition of PDGF receptor signaling in tumor stroma enhances antitumor effect of chemotherapy. Cancer Res 2002;62:5476-84.

52 Sethi T, Rintoul RC, Moore SM, et al. Extracellular matrix proteins protect small cell lung cancer cells against apoptosis: a mechanism for small cell lung cancer growth and drug resistance in vivo. Nat Med 1999:5:662-8.

53 Hynes RO. The extracellular matrix: not just pretty fibrils. Science 2009:326:1216-19.

$54 \mathrm{Ng} \mathrm{MR}$, Brugge JS. A stiff blow from the stroma: collagen crosslinking drives tumor progression. Cancer Cell 2009;16:455-7.

55 Courrech Staal EF, Wouters MW, van Sandick JW, et al. The stromal part of adenocarcinomas of the oesophagus: does it conceal targets for therapy? Eur J Cancer 2010:46:720-8.

56 Mesker WE, Junggeburt JM, Szuhai K, et al. The carcinoma-stromal ratio of colon carcinoma is an independent factor for survival compared to lymph node status and tumor stage. Cell Oncol 2007;29:387-98.

57 Josson S, Sharp S, Sung SY, et al. Tumor-stromal interactions influence radiation sensitivity in epithelial- versus mesenchymal-like prostate cancer cells. J Oncol 2010;2010:232831.

58 Muerkoster SS, Werbing V, Koch D, et al. Role of myofibroblasts in innate chemoresistance of pancreatic carcinoma-epigenetic downregulation of caspases. Int J Cancer 2008;123:1751-60.

59 Wu SD, Ma YS, Fang Y, et al. Role of the microenvironment in hepatocellular carcinoma development and progression. Cancer Treat Rev 2012;38: 218-25.

60 Hoshida Y, Villanueva A, Kobayashi M, et al. Gene expression in fixed tissues and outcome in hepatocellular carcinoma. N Engl J Med 2008;359: 1995-2004.

61 Finak G, Bertos N, Pepin F, et al. Stromal gene expression predicts clinical outcome in breast cancer. Nat Med 2008;14:518-27.

62 Garber K. Stromal depletion goes on trial in pancreatic cancer. J Natl Cancer Inst 2010;102:448-50.

63 Saadi A, Shannon NB, Lao-Sirieix P, et al. Stromal genes discriminate preinvasive from invasive disease, predict outcome, and highlight inflammatory pathways in digestive cancers. Proc Natl Acad Sci U S A 2010;107:2177-82.

64 Derynck R, Akhurst RJ, Balmain A. TGF-beta signaling in tumor suppression and cancer progression. Nat Genet 2001;29:117-29.

65 Achyut BR, Yang L. Transforming growth factor-beta in the gastrointestinal and hepatic tumor microenvironment. Gastroenterology 2011;141: 1167-78

66 Webber J, Steadman R, Mason MD, et al. Cancer exosomes trigger fibroblast to myofibroblast differentiation. Cancer Res 2010;70:9621-30.

67 Dunkern TR, Feurstein D, Rossi GA, et al. Inhibition of TGF-beta induced lung fibroblast to myofibroblast conversion by phosphodiesterase inhibiting drugs and activators of soluble guanylyl cyclase. Eur J Pharmacol 2007;572: $12-22$

68 Jain VK, Cunningham D, Chau I. Preoperative and postoperative chemotherapy for gastric cancer. Surg Oncol Clin N Am 2012;21:99-112.

69 Davies K. Malignant hyperthermia may be due to a defect in a large Ca2+ release channel protein. Trends Genet 1990;6:171-2. 